hep-th/0607131

\title{
't Hooft Expansion of 1/2 BPS Wilson Loop
}

\author{
Kazumi Okuyama \\ Department of Physics and Astronomy, University of British Columbia \\ Vancouver, BC, V6T 1Z1, Canada \\ kazumi@phas.ubc.ca
}

\begin{abstract}
We revisit the 't Hooft expansion of $1 / 2$ BPS circular Wilson loop in $\mathcal{N}=4$ SYM studied by Drukker and Gross in hep-th/0010274. We find an interesting recursion relation which relates different number of holes on the worldsheet. We also argue that we can turn on the string coupling by applying a certain integral transformation to the planar result.
\end{abstract}

July 2006 


\section{Introduction}

Even after the revolutions in string theory, we still lack enough control over the string coupling dependence of various amplitudes. Notable exceptions are the $c \leq 1$ non-critical strings and the topological strings. For the latter case, all genus results are available for some models. Moreover, there is a deep underlying structure in the genus expansion which enables us to determine the amplitudes recursively [1]. It is extremely interesting to find a similar relation in the non-topological setup, but in general it is a formidable task.

However, there exists an all order result in our familiar AdS/CFT context: the expectation value of $1 / 2$ BPS circular Wilson loop in $U(N) \mathcal{N}=4$ SYM [2]. In the string theory side, Wilson loop is described by a string worldsheet ending on the loop at the boundary of AdS, and its expectation value is given by the worldsheet action for the minimal surface [3, 4.5]. On the Yang-Mills side, 1/2 BPS circular Wilson loop has the form

$$
W=\left\langle\frac{1}{N} \operatorname{Tr} P \exp \left(\oint d t\left(i A_{\mu} \dot{x}^{\mu}+\theta^{i} \Phi_{i}|\dot{x}|\right)\right)\right\rangle
$$

where $\theta^{i}$ is a constant unit vector in $\mathbb{R}^{6}$ and $x^{\mu}(t)$ is a circular loop in $\mathbb{R}^{4}$. In a beautiful paper [6], it is realized that summing over the rainbow diagrams boil down to the following $N \times N$ Hermitian matrix model

$$
W=\frac{1}{Z} \int d M \exp \left(-\frac{2}{g_{\mathrm{YM}}^{2}} \operatorname{Tr} M^{2}\right) \frac{1}{N} \operatorname{Tr} e^{M} .
$$

In [2] it is further argued that this matrix model actually gives all order result of (1.1) in perturbation theory, up to possible instanton corrections [7].

In this paper, we will study the 't Hooft expansion [8] of circular Wilson loop

$$
W\left(\lambda, g_{s}\right)=\sum_{h, \ell=0}^{\infty} \mathcal{W}_{h, \ell} \lambda^{h} g_{s}^{2 \ell}
$$

in terms of the string coupling $g_{s} \sim g_{\mathrm{YM}}^{2}$ and the 't Hooft coupling $\lambda=g_{\mathrm{YM}}^{2} N$. As reviewed nicely in [9], a Feynman diagram of Yang-Mills theory is reorganized as a Riemann surface of $h$ holes and $\ell$ handles. Note that the $g_{s}$-expansion of Wilson loop starts with the order $g_{s}^{0}$, since the trace in (1.1) is normalized by $1 / N$. At least perturbatively, the double summation in (1.3) can be performed by either summing over $h$ first or summing over $\ell$ first. The first choice leads to the usual genus expansion of $W$. In section 2 , we will consider how to find the $\ell$-loop term systematically. The second choice leads to the expansion of 
$W$ in terms of the number of holes. In section 3, we will find a recursion relation between the $h$-hole amplitude and the $(h+1)$-hole amplitude. In both cases, we find a curious property of circular Wilson loop: we can turn on the coupling from zero-coupling by a certain operation:

$$
\begin{aligned}
& W\left(0, g_{s}\right) \longrightarrow W\left(\lambda, g_{s}\right) \\
& W(\lambda, 0) \longrightarrow W\left(\lambda, g_{s}\right) .
\end{aligned}
$$

In section 4 , we will study the relation between $W$ at $g_{s}=0$ and $g_{s} \neq 0$, and argue that they are related by an integral transformation. Section 5 is the discussions on our findings, and some technical details are collected in the appendices.

\section{Genus Expansion of Circular Wilson Loop}

In this section, we will consider the string loop expansion of circular Wilson loop

$$
W\left(\lambda, g_{s}\right)=\sum_{\ell=0}^{\infty} W_{\ell}(\lambda) g_{s}^{2 \ell}
$$

This problem has been already studied in [2]. However, we find it useful to revisit this problem since there is a systematics behind this which is not mentioned in [2].

\subsection{Relation between parameters in YM and String Theory}

Before starting the analysis, let us first review the relation between the parameters in $\mathcal{N}=4 \mathrm{SYM}$ and string theory on $A d S_{5} \times S^{5}[10,11$. The 't Hooft coupling $\lambda$ in $\mathcal{N}=4$ SYM corresponds to the curvature radius in the string theory side

$$
\left(R_{A d S_{5}}\right)^{2}=\left(R_{S^{5}}\right)^{2}=\alpha^{\prime} \sqrt{\lambda}
$$

In other words, $1 / \sqrt{\lambda}$ governs the worldsheet sigma-model corrections. The Yang-Mills coupling $g_{\mathrm{YM}}^{2}$ is related to the string coupling $g_{s}$ via the identification of complex coupling in $\mathcal{N}=4 \mathrm{SYM}$ and the axion-dilaton of Type IIB string

$$
\tau_{\mathrm{YM}}=\frac{\theta}{2 \pi}+i \frac{4 \pi}{g_{\mathrm{YM}}^{2}} \quad \Leftrightarrow \quad \tau_{\mathrm{IIB}}=\chi+i e^{-\phi} .
$$

In this paper, we define the string coupling $g_{s}$ as

$$
g_{s}=\frac{g_{\mathrm{YM}}^{2}}{4}
$$


This differs from the usual normalization by a factor of $\pi$. This factor can be absorbed by a constant shift of the dilaton zero-mode $\phi_{0}$. Our normalization is motivated by the fact that under the relation (2.4) the Gaussian measure of matrix model (1.2) becomes

$$
\int d M \exp \left(-\frac{2}{g_{\mathrm{YM}}^{2}} \operatorname{Tr} M^{2}\right)=\int d M \exp \left(-\frac{1}{2 g_{s}} \operatorname{Tr} M^{2}\right),
$$

which implies that $g_{s}$ with this normalization is the canonical loop counting parameter of the matrix model (2.5). In our normalization, 't Hooft coupling $\lambda=g_{\mathrm{YM}}^{2} N$ is written as

$$
\lambda=4 g_{s} N
$$

\subsection{Expansion in Terms of Buchholz Polynomials}

In [2], the matrix integral (1.2) was evaluated exactly at finite $N$ and the result is given by a Laguerre polynomial

$$
W=\frac{1}{N} e^{\frac{g_{s}}{2}} L_{N-1}^{1}\left(-g_{s}\right)
$$

This is also written as a confluent hypergeometric function

$$
W=e^{\frac{g_{s}}{2}}{ }_{1} F_{1}\left(1-N, 2 ;-g_{s}\right)=e^{-\frac{g_{s}}{2}}{ }_{1} F_{1}\left(1+N, 2 ; g_{s}\right)
$$

We would like to recast this into a form of (1.3) in the large $N$ limit with fixed $\lambda=4 g_{s} N$. The large $a$ behavior of confluent hypergeometric function ${ }_{1} F_{1}(a, b ; z)$ with general $b$ has been studied by mathematicians [12,13], so we can borrow their result and apply it to our case $b=2(2.8)$. The starting point of the analysis is the following contour integral representation of (2.7)

$$
W\left(\lambda, g_{s}\right)=2 \oint_{z=0} \frac{d z}{2 \pi i} \exp \left[\frac{\lambda}{2} z+\frac{g_{s}}{2} \operatorname{coth}\left(g_{s} z\right)\right] .
$$

The equivalence of (2.7) and (2.9) is shown in appendix A. Then we add and subtract the single pole of $\operatorname{coth}\left(g_{s} z\right)$ at $z=0$

$$
W\left(\lambda, g_{s}\right)=2 \oint_{z=0} \frac{d z}{2 \pi i} \exp \left[\frac{1}{2}\left(\lambda z+\frac{1}{z}\right)+g_{s} H\left(g_{s} z\right)\right],
$$

where $H(x)$ is defined by

$$
H(x)=\frac{1}{2}\left(\operatorname{coth} x-\frac{1}{x}\right)
$$


Since $H(x)$ is regular at $x=0$, the second factor in (2.10) has the Taylor expansion around $z=0$

$$
g_{s} H\left(g_{s} z\right)=\frac{g_{s}^{2}}{6} z-\frac{g_{s}^{4}}{90} z^{3}+\frac{g_{s}^{6}}{945} z^{5}-\frac{g_{s}^{8}}{9450} z^{7}+\frac{g_{s}^{10}}{93555} z^{9}+\cdots
$$

To evaluate the contour integral, we expand the integrand around $z=0$. Using the generating function of modified Bessel functions

$$
\exp \left[\frac{t}{2}\left(x+\frac{1}{x}\right)\right]=\sum_{n=-\infty}^{\infty} \frac{I_{n}(t)}{x^{n}}
$$

the first part in $(2.10)$ has the following Laurent expansion

$$
\exp \left[\frac{1}{2}\left(\lambda z+\frac{1}{z}\right)\right]=\sum_{k=-\infty}^{\infty} \frac{\widehat{I}_{k}(\lambda)}{z^{k}}
$$

where we introduced a function $\widehat{I}_{k}(\lambda)$ as

$$
\widehat{I}_{k}(\lambda)=\frac{I_{k}(\sqrt{\lambda})}{(\sqrt{\lambda})^{k}}
$$

The second factor of (2.10) is regular around $z=0$ and admits a Taylor expansion. The coefficient of $x^{n}$ in the Taylor expansion of $e^{a H(x)}$ is known as the Buchholz polynomial $p_{n}(a)[12,13]$

$$
\sum_{n=0}^{\infty} x^{n} p_{n}(a) \stackrel{\text { def }}{=} \exp [a H(x)] .
$$

Therefore, the second factor of (2.10) is expanded as

$$
\exp \left[g_{s} H\left(g_{s} z\right)\right]=\sum_{n=0}^{\infty}\left(g_{s} z\right)^{n} p_{n}\left(g_{s}\right)
$$

Finally, combining (2.14) and (2.17), the circular Wilson loop (2.10) is written as

$$
W\left(\lambda, g_{s}\right)=2 \sum_{n=0}^{\infty} \widehat{I}_{n+1}(\lambda) g_{s}^{n} p_{n}\left(g_{s}\right)
$$

From the definition (2.16), one can easily see that $p_{n}\left(g_{s}\right)$ is an $n^{\text {th }}$ order polynomial in $g_{s}$ with fixed parity $p_{n}\left(-g_{s}\right)=(-1)^{n} p_{n}\left(g_{s}\right)$. See appendix B for more information on $p_{n}\left(g_{s}\right)$. To our knowledge, the closed form of $p_{n}\left(g_{s}\right)$ is not known in the literature. 
However, starting from $p_{0}\left(g_{s}\right)=1$, we can generate $p_{n}\left(g_{s}\right)$ successively using the following recursion relation

$$
p_{n}\left(g_{s}\right)=g_{s} \int_{0}^{1} d t t^{\frac{n}{2}}\left[\frac{1}{4} p_{n-1}\left(g_{s} t\right)-p_{n-1}^{\prime \prime}\left(g_{s} t\right)\right]
$$

where $p_{n-1}^{\prime \prime}$ denotes the second derivative of $p_{n-1}$. For instance, the first few terms are given by

$$
p_{1}\left(g_{s}\right)=\frac{g_{s}}{6}, \quad p_{2}\left(g_{s}\right)=\frac{g_{s}^{2}}{72}, \quad p_{3}\left(g_{s}\right)=\frac{g_{s}^{3}}{1296}-\frac{g_{s}}{90}, \quad p_{4}\left(g_{s}\right)=\frac{g_{s}^{4}}{31104}-\frac{g_{s}^{2}}{540} .
$$

Although (2.18) is a nice compact expression, we have to rewrite it in the form of string loop expansion (2.1). This is basically a problem of the change of basis from the polynomials $\left\{g_{s}^{n} p_{n}\left(g_{s}\right)\right\}_{n=0}^{\infty}$ to the monomials $\left\{g_{s}^{2 \ell}\right\}_{\ell=0}^{\infty}$. We can easily find a first few terms of $\ell$-loop correction $W_{\ell}(\lambda)$ of Wilson loop using (2.20)

$$
\begin{aligned}
& W_{0}(\lambda)=2 \widehat{I}_{1}(\lambda) \\
& W_{1}(\lambda)=\frac{\widehat{I}_{2}(\lambda)}{3} \\
& W_{2}(\lambda)=-\frac{\widehat{I}_{4}(\lambda)}{45}+\frac{\widehat{I}_{3}(\lambda)}{36} \\
& W_{3}(\lambda)=\frac{2 \widehat{I}_{6}(\lambda)}{945}-\frac{\widehat{I}_{5}(\lambda)}{270}+\frac{\widehat{I}_{4}(\lambda)}{648} .
\end{aligned}
$$

In general, $W_{\ell}(\lambda)$ is a linear combination of $\widehat{I}_{k}(\lambda)$

$$
W_{\ell}(\lambda)=2 \sum_{m=0}^{\ell-1} c_{m} \widehat{I}_{\ell+m+1}(\lambda),
$$

where $c_{m}$ is the coefficient of $g_{s}^{\ell-m}$ in $p_{\ell+m}\left(g_{s}\right)$. Namely, $W_{\ell}(\lambda)$ is determined by certain coefficients in $p_{\ell}\left(g_{s}\right), p_{\ell+1}\left(g_{s}\right), \cdots, p_{2 \ell-1}\left(g_{s}\right)$. This implies that the string loop correction at fixed order $\ell$ is calculable by a finite number of steps using (2.19).

\subsection{Large $\lambda$ Limit}

From the expression (2.18), it is easy to find a leading $g_{s}$-correction to the large $\lambda$ behavior of Wilson loop. Recalling that the leading asymptotics of modified Bessel function $I_{k}(z)$ is independent of $k$

$$
I_{k}(z) \sim \frac{1}{\sqrt{2 \pi z}} e^{z} \quad(|z| \rightarrow \infty)
$$


(2.18) becomes

$$
\begin{aligned}
W\left(\lambda, g_{s}\right) & \sim \sqrt{\frac{2}{\pi}} \lambda^{-\frac{3}{4}} e^{\sqrt{\lambda}} \sum_{n=0}^{\infty} \frac{g_{s}^{n}}{(\sqrt{\lambda})^{n}} p_{n}\left(g_{s}\right) \\
& =\sqrt{\frac{2}{\pi}} \lambda^{-\frac{3}{4}} e^{\sqrt{\lambda}} \exp \left[g_{s} H\left(\frac{g_{s}}{\sqrt{\lambda}}\right)\right] .
\end{aligned}
$$

In the large $\lambda$ limit, the last factor is approximated by the first term in the expansion of $g_{s} H\left(g_{s} z\right)$ in (2.12). Therefore, we find

$$
W\left(\lambda, g_{s}\right) \sim \sqrt{\frac{2}{\pi}} \lambda^{-\frac{3}{4}} \exp \left(\sqrt{\lambda}+\frac{g_{s}^{2}}{6 \sqrt{\lambda}}\right) .
$$

This agrees with the result of [2] 10 Note that the expression (2.25) is valid in the regime

$$
\{\lambda \gg 1\} \cap\left\{\lambda \gg g_{s}^{2}\right\}
$$

In [2], it is argued that the $g_{s}$-correction found in (2.25) is understood from the consideration of worldsheet moduli integral in string theory on $A d S_{5} \times S^{5}$.

As argued in [14], when $g_{s}$ becomes of order $\sqrt{\lambda}$ the large $\lambda$ behavior of $W\left(\lambda, g_{s}\right)$ gets all order correction in $g_{s}^{2} / \lambda$ beyond the first order correction given in (2.25). In the bulk string theory side, this is computed by the DBI action of D3-brane [14]

$$
W\left(\lambda, g_{s}\right) \sim \exp \left[\frac{\lambda}{2 g_{s}} \sinh ^{-1}\left(\frac{g_{s}}{\sqrt{\lambda}}\right)+\frac{1}{2} \sqrt{\lambda+g_{s}^{2}}\right]
$$

In the Yang-Mills/matrix model side, this is easily obtained by the saddle point approximation of the contour integral (2.9). To reproduce this expression from the series expansion (2.18), we have to keep sub-leading terms in the large $\lambda$ asymptotics of modified Bessel function.

\section{Expansion in Terms of Number of Holes}

The 't Hooft expansion (1.3) is a double expansion in $h$ and $\ell$, thus we can try to sum over $\ell$ first and write $W$ in the form

$$
W=\sum_{h=0}^{\infty} N^{h} \mathcal{F}_{h}\left(g_{s}\right)
$$

\footnotetext{
1 To compare with the expression in [2], note that $g_{s}^{2} / 6=\left(g_{\mathrm{YM}}^{2}\right)^{2} / 96$.
} 
where $\mathcal{F}_{h}\left(g_{s}\right)$ is the amplitude with fixed $h$

$$
\mathcal{F}_{h}\left(g_{s}\right)=\left(4 g_{s}\right)^{h} \sum_{\ell=0}^{\infty} \mathcal{W}_{h, \ell} g_{s}^{2 \ell}
$$

For later convenience, we included the factor $\left(4 g_{s}\right)^{h}$ in $\lambda^{h}=\left(4 g_{s} N\right)^{h}$ into the definition of $\mathcal{F}_{h}\left(g_{s}\right)$. Usually we do not consider this form of expansion since it is not so illuminating. However, one advantage of this expansion is that we can study the analytic property of $\mathcal{F}_{h}\left(g_{s}\right)$ as a function of string coupling $g_{s}$, at least for fixed $h$. This may give a clue to understand the $g_{s}$-dependence of circular Wilson loop.

In order to write $W$ in the form (3.1), we use the second expression in (2.8) and write the confluent hypergeometric function as a summation

$$
\begin{aligned}
W & =e^{-\frac{g_{s}}{2}} \sum_{k=0}^{\infty} g_{s}^{k} \frac{(N+1)(N+2) \cdots(N+k)}{k !(k+1) !} \\
& =e^{-\frac{g_{s}}{2}} \sum_{k=0}^{\infty} \frac{g_{s}^{k}}{(k+1) !} \prod_{j=1}^{k}\left(1+\frac{N}{j}\right) .
\end{aligned}
$$

We would like to rewrite this as a power series in $N$.2 The zero-th order term is easily found by setting $N=0$ in $(3.3)$

$$
\mathcal{F}_{0}\left(g_{s}\right)=e^{-\frac{g_{s}}{2}} \sum_{k=0}^{\infty} \frac{g_{s}^{k}}{(k+1) !}=\frac{2}{g_{s}} \sinh \left(\frac{g_{s}}{2}\right) .
$$

The higher $h$ term has the form

$$
\mathcal{F}_{h}\left(g_{s}\right)=e^{-\frac{g_{s}}{2}} \sum_{k=0}^{\infty} \frac{g_{s}^{k}}{(k+1) !} H_{k}^{(h)}
$$

where the coefficient $H_{k}^{(h)}$ is determined recursively by

$$
H_{k}^{(0)}=1, \quad H_{k}^{(h)}=\sum_{n=1}^{k} \frac{1}{n} H_{n-1}^{(h-1)} .
$$

2 The $N$-dependent factor in the first line of (3.3) is known as the rising factorial. This can be expanded in terms of the Stirling number of the first kind $s(n, m)$

$$
(N+1)(N+2) \cdots(N+k)=\sum_{m=1}^{k} s(k+1, m+1)(-1)^{k-m} N^{m}
$$

However, this expression is not so useful for our purpose, so we will not use this. 
In particular, the coefficient in the $h=1$ term is the harmonic number: $H_{k}^{(1)}=\sum_{n=1}^{k} \frac{1}{n}$.

Remarkably, it turns out that $\mathcal{F}_{h}\left(g_{s}\right)$ and $\mathcal{F}_{h+1}\left(g_{s}\right)$ are related by the following integral transformation

$$
\mathcal{F}_{h+1}\left(g_{s}\right)=\int_{0}^{1} d t 2 \sinh \left(\frac{g_{s}}{2}(1-t)\right) \mathcal{F}_{h}\left(g_{s} t\right) .
$$

This is easily shown by expanding both sides in $g_{s}$ and using the relation (3.6). One can also write this relation (3.7) in the form of convolution

$$
\mathcal{F}_{h+1}\left(g_{s}\right)=\frac{1}{g_{s}}\left(\mathcal{B} * \mathcal{F}_{h}\right)\left(g_{s}\right)
$$

where $*$ is defined by

$$
(F * G)(x)=\int_{0}^{x} d y F(y) G(x-y) .
$$

The function $\mathcal{B}\left(g_{s}\right)$ appearing in (3.8) is given by

$$
\mathcal{B}\left(g_{s}\right)=2 \sinh \left(\frac{g_{s}}{2}\right)=g_{s} \mathcal{F}_{0}\left(g_{s}\right) .
$$

To summarize, one can increase the number of holes by one by taking a convolution with $\mathcal{B}\left(g_{s}\right)$, which is essentially given by the $h=0$ term.

As an illustration of the recursion relation, let us consider the behavior of $\mathcal{F}_{h}\left(g_{s}\right)$ near $g_{s}=0$. At the leading order in $g_{s}$, one can use the approximation $\mathcal{F}_{0} \sim 1$ and $\mathcal{B} \sim g_{s}$. Taking the convolution recursively, one can easily find

$$
\mathcal{F}_{h}\left(g_{s}\right)=\frac{g_{s}^{h}}{h !(h+1) !}+\mathcal{O}\left(g_{s}^{h+2}\right)
$$

Plugging this into (3.1), we recover the planar result $W_{0}(\lambda)=2 \widehat{I_{1}}(\lambda)$.

Our relation (3.8) is not limited to the perturbative regime in $g_{s}$. We are able to talk about the analytic property of $\mathcal{F}_{h}\left(g_{s}\right)$. For $h=0$, it is clear that $\mathcal{F}_{0}\left(g_{s}\right)$ given in (3.4) is analytic on the whole $g_{s}$-plane. Let us look at the next term $h=1$. Setting $h=0$ in (3.7) and evaluating the integral, we find

$$
\mathcal{F}_{1}\left(g_{s}\right)=\frac{1}{g_{s}}\left[e^{\frac{g_{s}}{2}} \operatorname{Ein}\left(g_{s}\right)+e^{-\frac{g_{s}}{2}} \operatorname{Ein}\left(-g_{s}\right)\right]
$$

where $\operatorname{Ein}(x)$ denotes the entire exponential integral ${ }^{3}$

$$
\operatorname{Ein}(x)=\int_{0}^{x} \frac{d t}{t}\left(1-e^{-t}\right)=\sum_{k=1}^{\infty} \frac{(-1)^{k-1} x^{k}}{k ! k} .
$$

3 The entire exponential integral is related to the ordinary exponential integral by

$$
\mathrm{E}_{1}(x)=\int_{x}^{\infty} \frac{d t}{t} e^{-t}=-\gamma-\log x+\operatorname{Ein}(x) .
$$


Note that $\mathcal{F}_{1}\left(g_{s}\right)$ is regular at $g_{s}=0$. The apparent singularity at $g_{s}=0$ due to the overall factor $1 / g_{s}$ in (3.12) is canceled by the function inside the bracket which behaves as $g_{s}^{2}$ near $g_{s}=0$. Therefore, $\mathcal{F}_{1}\left(g_{s}\right)$ vanishes linearly as $g_{s} \rightarrow 0$, as expected from the general form (3.5). Actually $\mathcal{F}_{1}\left(g_{s}\right)$ is an entire function of $g_{s}$, since $\operatorname{Ein}\left(g_{s}\right)$ is entire as the name suggests. By induction, one can argue that $\mathcal{F}_{h}\left(g_{s}\right)$ are analytic for all $h$, since the integral (3.7) is always convergent and there is no source of singularity.

At least formally, our relation (3.8) suggests that starting from $\lambda=0$

$$
\mathcal{F}_{0}\left(g_{s}\right)=W\left(\lambda=0, g_{s}\right),
$$

we can turn on $\lambda$ by successively applying the convolution

$$
W\left(\lambda, g_{s}\right)=\sum_{h=0}^{\infty}\left(\frac{\lambda}{4 g_{s}}\right)^{h}\left[\frac{1}{g_{s}}\left(g_{s} \mathcal{F}_{0}\right) *\right]^{h} \mathcal{F}_{0}\left(g_{s}\right)
$$

In the next section, we will find a relation between $g_{s}=0$ and $g_{s} \neq 0$.

\section{Turning on $g_{s}$ from $g_{s}=0$}

\subsection{Formal Expression of $W$}

To see the relation between $g_{s}=0$ and $g_{s} \neq 0$, let us start with the contour integral representation of $W_{0}(\lambda)=W\left(\lambda, g_{s}=0\right)$

$$
W_{0}(\lambda)=2 \oint_{z=0} \frac{d z}{2 \pi i} e^{\frac{1}{2}\left(\lambda z+\frac{1}{z}\right)}=2 \widehat{I}_{1}(\lambda) .
$$

The key observation is that the differentiation of $W_{0}(\lambda)$ by $\lambda$ is equivalent to the insertion of $z$ in the contour integral

$$
2 \frac{\partial}{\partial \lambda} \leftrightarrow z
$$

By looking at the contour integral representation of $W\left(\lambda, g_{s}\right)$ in (2.10), one immediately notices that

$$
W\left(\lambda, g_{s}\right)=\exp \left[g_{s} H\left(2 g_{s} \frac{\partial}{\partial \lambda}\right)\right] W_{0}(\lambda) .
$$

This relation can be also obtained from the expansion in terms of $p_{n}\left(g_{s}\right)$ (2.18). From (2.14), $\widehat{I}_{k}(\lambda)$ is given by

$$
\widehat{I}_{k}(\lambda)=\oint_{z=0} \frac{d z}{2 \pi i} z^{k-1} e^{\frac{1}{2}\left(\lambda z+\frac{1}{z}\right)} .
$$


Again, using the correspondence (4.2), one finds that the derivative with respect to $\lambda$ increases the index of $\widehat{I}_{k}(\lambda)$

$$
\left(2 \frac{\partial}{\partial \lambda}\right)^{n} \widehat{I}_{k}(\lambda)=\widehat{I}_{k+n}(\lambda) .
$$

Using this, we can rewrite $(2.18)$ as

$$
W\left(\lambda, g_{s}\right)=2 \sum_{n=0}^{\infty}\left(2 g_{s} \frac{\partial}{\partial \lambda}\right)^{n} p_{n}\left(g_{s}\right) \widehat{I}_{1}(\lambda)
$$

which is equivalent to (4.3) from the definition of $p_{n}(2.16)$.

To summarize, we found a remarkable property of $1 / 2$ BPS circular Wilson loop: turning on the string coupling from zero coupling amounts to acting a certain differential operator of $\lambda$ on the planar result $W_{0}(\lambda)$.

Although (4.3) is elegant, it is still a formal expression. In particular, the differential operator appearing in (4.3) involves a derivative $\partial_{\lambda}^{n}$ with arbitrarily large $n$. Therefore, it might be better to interpret it as an integral transform rather than a differential operator. In the next subsection, we will consider the Chern-Simons theory on $S^{3}$ as a simple example to see if this interpretation works.

\subsection{Digression to the Chern-Simons Theory on $S^{3}$}

The partition function of $S U(N)$ Chern-Simons theory on $S^{3}$ is exactly known [15], and its 't Hooft expansion was studied in [16]. Via a geometric transition, this is equivalent to the topological A-model on the resolved conifold. The Kähler parameter of $\mathbb{P}^{1}$ is identified as the 't Hooft coupling $t=g_{s} N$ of Chern-Simons theory. The partition function looks like this:

$$
Z_{\text {conifold }}\left(t, g_{s}\right)=Z_{\text {point }}\left(g_{s}\right) Z_{\text {pert }}\left(t, g_{s}\right)
$$

where

$$
\begin{aligned}
Z_{\text {point }}\left(g_{s}\right) & =\prod_{n=1}^{\infty}\left(1-e^{-n g_{s}}\right)^{-n} \\
Z_{\text {pert }}\left(t, g_{s}\right) & =\prod_{n=1}^{\infty}\left(1-e^{-t-n g_{s}}\right)^{n} .
\end{aligned}
$$

We are interested in the $t$-dependent part $Z_{\text {pert }}\left(t, g_{s}\right)$. The free energy has the following genus expansion

$$
\log Z_{\text {pert }}\left(t, g_{s}\right)=\frac{1}{g_{s}^{2}} F_{0}(t)-\frac{t}{24}+F\left(t, g_{s}\right)
$$


The first term is the genus zero term, the second term is a part of the genus one term which is linear in $t$, and the last term is a sum of $g \geq 2$ terms and a remaining part of $g=1$ term

$$
F\left(t, g_{s}\right)=\sum_{g=1}^{\infty} g_{s}^{2 g-2} \frac{B_{2 g}}{2 g(2 g-2) !} \mathrm{Li}_{3-2 g}\left(e^{-t}\right) .
$$

Here $\operatorname{Li}_{k}(z)$ denotes the polylogarithm

$$
\operatorname{Li}_{k}(z)=\sum_{n=1}^{\infty} \frac{z^{n}}{n^{k}}
$$

From this definition, it follows that the function of $t$ appearing in the free energy (4.10) satisfies

$$
\left(-\frac{\partial}{\partial t}\right)^{n} \operatorname{Li}_{k}\left(e^{-t}\right)=\operatorname{Li}_{k-n}\left(e^{-t}\right)
$$

One can immediately notice a similarity of this relation and (4.5) for $\widehat{I}_{k}(\lambda)$. From (4.12), we can rewrite $(4.10)$ as

$$
F\left(t, g_{s}\right)=\sum_{g=1}^{\infty} \frac{B_{2 g}}{2 g(2 g-2) !}\left(g_{s} \frac{\partial}{\partial t}\right)^{2 g-2} \operatorname{Li}_{1}\left(e^{-t}\right) \equiv 12 K\left(g_{s} \frac{\partial}{\partial t}\right) F_{1}^{\text {inst }}(t) \text {. }
$$

Here we introduced a function $K(x)$

$$
K(x)=\sum_{g=1}^{\infty} \frac{B_{2 g}}{2 g(2 g-2) !} x^{2 g-2},
$$

and a part of the genus one free energy written as a sum of worldsheet instantons

$$
F_{1}^{\text {inst }}(t)=\frac{1}{12} \operatorname{Li}_{1}\left(e^{-t}\right)=-\frac{1}{12} \log \left(1-e^{-t}\right) .
$$

(4.13) means that all loop free energy is obtained from the $g=1$ term by acting a differential operator of 't Hooft coupling.

Again, the differential operator in (4.13) is of infinite order. One might try to define it by using the Fourier transform of $K(x)$

$$
K(x)=\int d p \widetilde{K}(p) e^{-i x p}
$$

Then the action of $K\left(g_{s} \partial_{t}\right)$ in (4.13) is given by a shift of $t$

$$
F\left(t, g_{s}\right)=12 \int d p \widetilde{K}(p) F_{1}^{\mathrm{inst}}\left(t-i g_{s} p\right)
$$


Unfortunately, it turns out that $K(x)$ is not Fourier-transformable. However, there exists an expression like (4.16) with the integration region restricted to the positive real axis $0 \leq p \leq \infty$. To see this, we use the integral representation of Bernoulli number

$$
\int_{0}^{\infty} d p \frac{2 p^{2 g-1}}{e^{2 \pi p}-1}=(-1)^{g-1} \frac{B_{2 g}}{2 g} .
$$

Plugging this into (4.14), $K(x)$ becomes

$$
\begin{aligned}
K(x) & =\sum_{g=1}^{\infty} \int_{0}^{\infty} d p \frac{2 p^{2 g-1}}{e^{2 \pi p}-1}(-1)^{g-1} \frac{1}{(2 g-2) !} x^{2 g-2} \\
& =\int_{0}^{\infty} d p \frac{2 p}{e^{2 \pi p}-1} \cos (x p) .
\end{aligned}
$$

Finally, we arrive at an integral form of (4.13)

$$
F\left(t, g_{s}\right)=12 \int_{0}^{\infty} d p \frac{p}{e^{2 \pi p}-1}\left[F_{1}^{\mathrm{inst}}\left(t+i g_{s} p\right)+F_{1}^{\mathrm{inst}}\left(t-i g_{s} p\right)\right]
$$

The failure of the existence of Fourier transform of $K(x)$ is seen by writing (4.19) as

$$
K(x)=\int_{0}^{\infty} d p\left(p \operatorname{coth}(\pi p) \cos (x p)-\frac{d}{d p} \sin (x p)\right) .
$$

The second term cannot be dropped since it oscillates at the upper end $p=\infty$. Let us ignore this term for the moment. The first term in (4.21) can be extended to the whole $p$-axis

$$
K(x) \sim \frac{1}{2} \int_{-\infty}^{\infty} d p p \operatorname{coth}(\pi p) e^{-i x p} .
$$

If we use this expression in (4.13), we get

$$
F\left(t, g_{s}\right) \sim 6 \int_{-\infty}^{\infty} d p p \operatorname{coth}(\pi p) F_{1}^{\text {inst }}\left(t-i g_{s} p\right) .
$$

By closing the contour of $p$-integral and picking up the residues at $p=i n(n=1,2, \cdots)$, we find

$$
F\left(t, g_{s}\right) \sim-12 \sum_{n=1}^{\infty} n F_{1}^{\mathrm{inst}}\left(t+g_{s} n\right)=\sum_{n=1}^{\infty} n \log \left(1-e^{-t-n g_{s}}\right)
$$

which is exactly the $\log$ of $Z_{\text {pert }}\left(t, g_{s}\right)$ in the infinite product form (4.8). 


\subsection{Integral Transformation of $W_{0}(\lambda)$ to $W\left(\lambda, g_{s}\right)$}

Let us return to the circular Wilson loop case. In (4.3), we found that $g_{s}=0$ and $g_{s} \neq 0$ are related by

$$
W\left(\lambda, g_{s}\right)=G\left(2 g_{s} \partial_{\lambda}, g_{s}\right) W_{0}(\lambda)
$$

where $G\left(x, g_{s}\right)=e^{g_{s} H(x)}$. As in the previous subsection, we will try to write it in the form of Fourier integral

$$
G\left(x, g_{s}\right)=\int d p \widetilde{G}\left(p, g_{s}\right) e^{-i x p} .
$$

The Chern-Simons example suggests that the region of $p$-integral does not necessarily extend to the whole $p$-axis. Then (4.25) is written as an integral transform

$$
W\left(\lambda, g_{s}\right)=\int d p \widetilde{G}\left(p, g_{s}\right) W_{0}\left(\lambda-2 i g_{s} p\right) .
$$

Note that the integral kernel $\widetilde{G}\left(p, g_{s}\right)$ has an explicit $g_{s}$-dependence whereas $\widetilde{K}(p)$ in the Chern-Simons case does not.

Unfortunately, we do not know how to compute the kernel $\widetilde{G}\left(p, g_{s}\right)$ in a closed form. Therefore, in the following we will construct the kernel perturbatively in $g_{s}$. By expanding $e^{g_{s} H(x)}$ as a power series in $g_{s}$

$$
e^{g_{s} H(x)}=\sum_{n=0}^{\infty} \frac{g_{s}^{n}}{n !} H(x)^{n} .
$$

we will rewrite $H(x)^{n}$ into the form (4.16) term by term. From the explicit calculation of lower order terms, we conjecture that $H(x)^{n}$ has the following integral representation depending on the parity of $n$ :

$$
\begin{aligned}
& H(x)^{2 k-1}=\int_{0}^{\infty} d p \sin (x p) G_{2 k-1}(p), \\
& H(x)^{2 k}=\int_{0}^{\infty} d p(1-\cos (x p)) G_{2 k}(p) .
\end{aligned}
$$

Then (4.25) is written in a form of integral transform

$$
W\left(\lambda, g_{s}\right)-W_{0}(\lambda)=\int_{0}^{\infty} d p\left[G_{-}\left(p, g_{s}\right) W_{-}\left(\lambda, g_{s} p\right)+G_{+}\left(p, g_{s}\right) W_{+}\left(\lambda, g_{s} p\right)\right]
$$

where we defined

$$
\begin{aligned}
& W_{-}\left(\lambda, g_{s} p\right)=\frac{W_{0}\left(\lambda+2 i g_{s} p\right)-W_{0}\left(\lambda-2 i g_{s} p\right)}{2 i} \\
& W_{+}\left(\lambda, g_{s} p\right)=W_{0}(\lambda)-\frac{W_{0}\left(\lambda+2 i g_{s} p\right)+W_{0}\left(\lambda-2 i g_{s} p\right)}{2} .
\end{aligned}
$$


and

$$
\begin{aligned}
& G_{-}\left(p, g_{s}\right)=\sum_{k=1}^{\infty} \frac{g_{s}^{2 k-1}}{(2 k-1) !} G_{2 k-1}(p) \\
& G_{+}\left(p, g_{s}\right)=\sum_{k=1}^{\infty} \frac{g_{s}^{2 k}}{(2 k) !} G_{2 k}(p) .
\end{aligned}
$$

In appendix $\mathrm{C}$, we computed the integral kernel of $H(x)^{n}$ for first few orders. The result is

$$
\begin{aligned}
& G_{1}(p)=\operatorname{Li}_{0}\left(e^{-\pi p}\right) \\
& G_{2}(p)= \frac{1}{2} p \operatorname{Li}_{0}\left(e^{-\pi p}\right)+\frac{\operatorname{Li}_{1}\left(e^{-\pi p}\right)}{\pi} \\
& G_{3}(p)=\frac{1}{4}\left[\left(1-\frac{1}{2} p^{2}\right) \operatorname{Li}_{0}\left(e^{-\pi p}\right)-3 p \frac{\operatorname{Li}_{1}\left(e^{-\pi p}\right)}{\pi}-6 \frac{\operatorname{Li}_{2}\left(e^{-\pi p}\right)}{\pi^{2}}\right] \\
& G_{4}(p)=\frac{1}{3 !}\left[\left(p-\frac{p^{3}}{8}\right) \operatorname{Li}_{0}\left(e^{-\pi p}\right)+3\left(1-\frac{1}{2} p^{2}\right) \frac{\operatorname{Li}_{1}\left(e^{-\pi p}\right)}{\pi}\right. \\
&\left.-\left(3+\frac{11 p}{2}\right) \frac{\operatorname{Li}_{2}\left(e^{-\pi p}\right)}{\pi^{2}}-16 \frac{\operatorname{Li}_{3}\left(e^{-\pi p}\right)}{\pi^{3}}\right]
\end{aligned}
$$

Let us make a few comments on the properties of kernel $G_{ \pm}\left(p, g_{s}\right)$. As in the ChernSimons case, the kernel has poles on the imaginary $p$-axis. Although we cannot close the contour of $p$-integral, those poles are intimately related to the analytic property of $W\left(\lambda, g_{s}\right)$ as a function of $g_{s}$. One can observe that the lower order terms (4.33) contains a piece

$$
\operatorname{Li}_{0}\left(e^{-\pi p}\right)=\frac{1}{e^{\pi p}-1}
$$

which has poles at

$$
p_{\text {pole }}=2 \text { in } \quad n \in \mathbb{Z}
$$

At this pole, the shifted argument of $W_{0}(\lambda)$ in (4.31) is given by

$$
\lambda \mp 2 i g_{s} p_{\text {pole }}=\lambda \pm 4 g_{s} n=4 g_{s}(N \pm n) .
$$

Namely, the shift of $\lambda$ at $p_{\text {pole }}$ is equivalent to an integer shift of $N$. This was also the case for the Chern-Simons theory. We speculate that the existence of the poles at these points reflects the underlying discreteness of $\lambda / 4 g_{s}$. 


\section{Discussions}

In this paper, we studied the 't Hooft expansion of 1/2 BPS circular Wilson loop $W\left(\lambda, g_{s}\right)$ in $\mathcal{N}=4 \mathrm{SYM}$ and found its curious properties.

First, we found an operation which increases the number of holes by one. We do not understand the physical meaning of it. It would be nice to find a physical origin of this relation.

Second, we found that $W\left(\lambda, g_{s}\right)$ is obtained by acting a differential operator of $\lambda$ on $W(\lambda, 0)$. We also observed a similar relation for the free energy $F\left(t, g_{s}\right)$ of Chern-Simons theory on $S^{3}$. It might be the case that the similarity of 't Hooft expansion of circular Wilson loop and Chern-Simons free energy merely means that 1/2 BPS Wilson loop is essentially a topological object. Indeed, it is argued in [2] that the whole dependence of circular Wilson loop on $\lambda$ and $g_{s}$ is coming from the anomaly of conformal transformation from straight line to circular loop. We also suspect that this similarity is a consequence of a general property of matrix model, since both $W\left(\lambda, g_{s}\right)$ [6,2] and $F\left(t, g_{s}\right)$ [17, 18] have matrix model representation. In both cases, the amplitudes have the structure

$$
A\left(t, g_{s}\right)=\sum_{n} g_{s}^{n} f_{n}\left(g_{s}\right) A_{n}(t), \quad \partial_{t}^{k} A_{n}(t)=A_{n+k}(t)
$$

For the Chern-Simons case, $f_{n}\left(g_{s}\right)=\frac{1+(-1)^{n}}{2} \chi_{n}$ with constant $\chi_{n}$. It is interesting to see if this structure appears in other cases.

Besides similarity, there are some differences between $W\left(\lambda, g_{s}\right)$ and $F\left(t, g_{s}\right)$. The main source of difference is the 't Hooft coupling dependence of worldsheet instanton action:

$$
\begin{aligned}
& \exp \left(-S_{\text {inst }}\right)=\exp (\sqrt{\lambda}) \quad \text { string on } \mathrm{AdS} / \mathcal{N}=4 \mathrm{SYM} \\
& \exp \left(-S_{\text {inst }}\right)=\exp (-t) \quad: \text { topological string/Chern-Simons theory }
\end{aligned}
$$

For the AdS case, by $S_{\text {inst }}$ we mean a regularized worldsheet action for the minimal surface [5]. Since the $g_{s} \neq 0$ result is obtained by acting $G\left(2 g_{s} \partial_{\lambda}, g_{s}\right)$ or $K\left(g_{s} \partial_{t}\right)$ to the $g_{s}=0$ value, the $g_{s}$-dependence is closely related to the 't Hooft coupling dependence of planar result. In particular, the existence of $q$-expansion with $q=e^{-g_{s}}$ is related to the linear dependence of worldsheet instanton action on 't Hooft coupling. Because of the form of the instanton action $e^{\sqrt{\lambda}}$, the circular Wilson loop does not have a $q$-expansion as we saw in the text. 


\section{Appendix A. Contour Integral Representation of $W$}

In this appendix, we will show that the contour integral in (2.9) is equal to the Laguerre polynomial expression of Wilson loop (2.7). First, we rescale the variable as $z \rightarrow z / 2 g_{s}$. Then the integral (2.9) becomes

$$
\begin{aligned}
W & =\frac{1}{g_{s}} \oint_{z=0} \frac{d z}{2 \pi i} \exp \left(N z+\frac{g_{s}}{2} \operatorname{coth} \frac{z}{2}\right) \\
& =\frac{1}{g_{s}} e^{\frac{g_{s}}{2}} \oint_{z=0} \frac{d z}{2 \pi i} \exp \left(N z+\frac{g_{s}}{e^{z}-1}\right) .
\end{aligned}
$$

Here we used $\lambda=4 g_{s} N$. By the change of variable $e^{z}=1+w$, this is rewritten as

$$
\begin{aligned}
W & =\frac{1}{g_{s}} e^{\frac{g_{s}}{2}} \oint_{w=0} \frac{d w}{2 \pi i}(1+w)^{N-1} e^{\frac{g_{s}}{w}} \\
& =\frac{1}{g_{s}} e^{\frac{g_{s}}{2}} \oint_{w=0} \frac{d w}{2 \pi i} \sum_{k=0}^{N-1}\left(\begin{array}{c}
N-1 \\
k
\end{array}\right) w^{k} \sum_{n=0}^{\infty} \frac{g_{s}^{n}}{n ! w^{n}} \\
& =e^{\frac{g_{s}}{2}} \sum_{k=0}^{N-1}\left(\begin{array}{c}
N-1 \\
k
\end{array}\right) \frac{g_{s}^{k}}{(k+1) !} \\
& =\frac{1}{N} e^{\frac{g_{s}}{2}} \sum_{k=0}^{N-1}\left(\begin{array}{c}
N \\
k+1
\end{array}\right) \frac{g_{s}^{k}}{k !} .
\end{aligned}
$$

Recalling the definition of Laguerre polynomial

$$
L_{N-1}^{1}(x)=\sum_{k=0}^{N-1}\left(\begin{array}{c}
N \\
k+1
\end{array}\right) \frac{(-x)^{k}}{k !},
$$

we can see that the last expression of (A.2) is equal to the matrix model result (2.7).

\section{Appendix B. Some Properties of Buchholz Polynomials}

In this appendix, we summarize some useful properties of Buchholz polynomials $p_{n}(a)$, which are defined by

$$
\exp [a H(x)]=\sum_{n=0}^{\infty} p_{n}(a) x^{n}
$$

where $H(x)$ is

$$
H(x)=\frac{1}{2}\left(\operatorname{coth} x-\frac{1}{x}\right)=\sum_{n=1}^{\infty} \frac{x}{x^{2}+\pi^{2} n^{2}} .
$$


Expanding the last expression around $x=0$, we find that the Taylor coefficient $b_{k}$ of $H(x)$

$$
H(x)=\sum_{k=1}^{\infty} b_{k} x^{2 k-1}
$$

is given by the Riemann zeta function (or equivalently by the Bernoulli number)

$$
b_{k}=(-1)^{k-1} \frac{\zeta(2 k)}{\pi^{2 k}}=\frac{2^{2 k-1} B_{2 k}}{(2 k) !} .
$$

Let us summarize some properties of $p_{n}(a)$ which follow directly from the definition (B.1). From the obvious relation $e^{(a+b) H}=e^{a H} e^{b H}$, it follows that

$$
p_{n}(a+b)=\sum_{k=0}^{n} p_{k}(a) p_{n-k}(b) .
$$

Next, by taking the $x$-derivative on both sides of (B.1) and using (B.3), we find a recursion relation

$$
p_{n}(a)=\frac{a}{n} \sum_{k=1}^{\left[\frac{n+1}{2}\right]}(2 k-1) b_{k} p_{n+1-2 k}(a) .
$$

This relation states that the $n^{\text {th }}$ polynomial $p_{n}(a)$ is determined by the lower order polynomials $p_{k}(a)(k<n)$. Although this relation determines $p_{n}(a)$ recursively, it involves many terms. A simpler relation between the consecutive neighbors $p_{n}(a)$ and $p_{n-1}(a)$ can be found as follows. From the definition (B.2), $H(x)$ satisfies the relation

$$
\frac{1}{2} \frac{d}{d x} H(x)+\frac{1}{x} H(x)=\frac{1}{4}-H(x)^{2} .
$$

This implies a differential equation for $e^{a H(x)}$

$$
\left(\frac{1}{2 a} \frac{\partial}{\partial x}+\frac{1}{x} \frac{\partial}{\partial a}\right) e^{a H(x)}=\left(\frac{1}{4}-\frac{\partial^{2}}{\partial a^{2}}\right) e^{a H(x)},
$$

which is equivalent to the following relation between $p_{n}(a)$ and $p_{n-1}(a)$

$$
\frac{n}{2 a} p_{n}(a)+p_{n}^{\prime}(a)=\frac{1}{4} p_{n-1}(a)-p_{n-1}^{\prime \prime}(a) .
$$

One can easily integrate this equation and find the integral form of this relation in the text (2.19).

The closed form expression of $p_{n}(a)$ is not known in the literature. However, we can find some coefficients of higher or lower powers of $a$. The highest power term $a^{n}$ of $p_{n}(a)$ 
is determined by the first term $b_{1} x=x / 6$ in the expansion of $H(x)$ (B.3). Expanding the exponential $e^{a x / 6}$, we find

$$
p_{n}(a)=\frac{a^{n}}{6^{n} n !}+\text { (lower order terms) } .
$$

Next, let us consider the lower order terms. From the expansion of $e^{a H(x)}$ to the first order in $a$

$$
e^{a H(x)}=1+a \sum_{k=1}^{\infty} b_{k} x^{2 k-1}+\cdots,
$$

it follows that the odd-order polynomial $p_{2 k-1}(a)$ starts with the term $b_{k} a$. We can then fix the coefficient of $a^{2}$ in the even-degree polynomial $p_{2 k}(a)$ by using the recursion relation (B.9). Setting $n=2 k+1$ in (B.9) and comparing the $\mathcal{O}\left(a^{0}\right)$ terms on both sides, we find that $p_{2 k}(a)$ starts with the term $-\frac{2 k+3}{4} b_{k+1} a^{2}$. We can repeat this process and find some lower order terms as

$$
\begin{aligned}
& p_{2 k-1}(a)=b_{k} a+\frac{1}{4 \cdot 3 !}\left[\frac{1}{2 !}(2 k+3)(2 k+4) b_{k+1}+b_{k}\right] a^{3}+\cdots, \\
& p_{2 k}(a)=-\frac{2 k+3}{4} b_{k+1} a^{2} \\
& \quad-\frac{1}{3 ! 4 !}\left[\frac{1}{2^{3}}(2 k+5)(2 k+6)(2 k+7) b_{k+2}+(2 k+4) b_{k+1}\right] a^{4}+\cdots .
\end{aligned}
$$

There is an alternative, more direct way to fix the coefficients of $p_{n}(a)$. From the expansion

$$
\exp [a H(x)]=\sum_{k=0}^{\infty} \frac{a^{k}}{k !} H(x)^{k}=\sum_{n=0}^{\infty} x^{n} p_{n}(a),
$$

the coefficients of $a^{k}$ in $p_{n}(a)$ is given by the Taylor coefficient of $x^{n}$ in $H(x)^{k} / k$ !. Therefore, all we need to know is the Taylor expansion of $H(x)^{k}$. For example, let us consider $H(x)^{2}$. Using the last expression in (B.2) $H(x)^{2}$ is written as

$$
\begin{aligned}
H(x)^{2} & =x^{2} \sum_{n=1}^{\infty} \sum_{m=1}^{\infty} \frac{1}{\left(x^{2}+\pi^{2} n^{2}\right)\left(x^{2}+\pi^{2} m^{2}\right)} \\
& =x^{2} \sum_{n=1}^{\infty} \frac{1}{\left(x^{2}+\pi^{2} n^{2}\right)^{2}}+x^{2} \sum_{n \neq m} \frac{1}{\left(x^{2}+\pi^{2} n^{2}\right)\left(x^{2}+\pi^{2} m^{2}\right)} \\
& =x^{2} \sum_{n=1}^{\infty} \frac{1}{\left(x^{2}+\pi^{2} n^{2}\right)^{2}}+x^{2} \sum_{n \neq m}\left(\frac{1}{x^{2}+\pi^{2} n^{2}}-\frac{1}{x^{2}+\pi^{2} m^{2}}\right) \frac{1}{\pi^{2}\left(m^{2}-n^{2}\right)} \\
& =x^{2} \sum_{n=1}^{\infty} \frac{1}{\left(x^{2}+\pi^{2} n^{2}\right)^{2}}+x^{2} \sum_{n=1}^{\infty} \frac{1}{x^{2}+\pi^{2} n^{2}} \frac{3}{2 \pi^{2} n^{2}} .
\end{aligned}
$$


In the last step, we used the relation

$$
\sum_{m \geq 1, m \neq n} \frac{1}{m^{2}-n^{2}}=\frac{3}{4 n^{2}} .
$$

Finally, expanding the last expression in (B.14), we reproduce the coefficient of $a^{2}$ in $p_{2 k}(a)$ obtained in (B.12) by the recursion relation. As one can easily see, this approach becomes extremely cumbersome as the power of $H(x)^{k}$ increases.

\section{Appendix C. Integral Representation of $H(x)^{k}$}

In this appendix, we will rewrite $H(x)^{k}$ as a Fourier-like integral.

Let us start with the $k=1$ case. Using the expansion of $H(x)(\mathbb{B} .3)$ with coefficient given in $(\mathbb{B . 4})$, we find

$$
\begin{aligned}
H(x) & =\sum_{k=1}^{\infty}(-1)^{k-1} \sum_{n=1}^{\infty} \frac{1}{(\pi n)^{2 k}} x^{2 k-1} \\
& =\int_{0}^{\infty} d p \sum_{k=1}^{\infty}(-1)^{k-1} \frac{t^{2 k-1}}{(2 k-1) !} x^{2 k-1} \sum_{n=1}^{\infty} e^{-\pi n p} \\
& =\int_{0}^{\infty} d p \sin (x p) \operatorname{Li}_{0}\left(e^{-\pi p}\right) .
\end{aligned}
$$

Next consider $k=2$. The Taylor coefficient of $x^{2 k}$ in $H(x)^{2} / 2$ ! is the coefficient of $a^{2}$ in $p_{2 k}(a)(\mathrm{B} .12)$. Therefore, we find

$$
\begin{aligned}
H(x)^{2} & =-\frac{1}{2} \sum_{k=1}^{\infty}(2 k+3) b_{k+1} x^{2 k} \\
& =\frac{1}{2} \sum_{k=1}^{\infty}(-1)^{k-1}(2 k+3) \sum_{n=1}^{\infty} \frac{1}{(\pi n)^{2 k+2}} x^{2 k} \\
& =\int_{0}^{\infty} d p \sum_{k=1}^{\infty}(-1)^{k-1}\left[\frac{2 k+1}{2} \frac{p^{2 k+1}}{(2 k+1) !} \sum_{n=1}^{\infty} e^{-\pi n p}+\frac{p^{2 k}}{(2 k) !} \sum_{n=1}^{\infty} \frac{1}{\pi n} e^{-\pi n p}\right] x^{2 k} \\
& =\int_{0}^{\infty} d p(1-\cos (x p))\left[\frac{1}{2} p \operatorname{Li}_{0}\left(e^{-\pi p}\right)+\frac{1}{\pi} \operatorname{Li}_{1}\left(e^{-\pi p}\right)\right] .
\end{aligned}
$$

In the third step, we split $2 k+3$ into $(2 k+1)+2$ and introduced the $p$-integral with different power, $p^{2 k+1}$ or $p^{2 k}$.

The computation of the higher power of $H(x)$ is similar (but tedious). We can find the Taylor coefficient of $H(x)^{n} / n$ ! by looking at the $a^{n}$ term in (B.12). Using the coefficients in (B.12), we obtain the kernel of $H(x)^{3}$ and $H(x)^{4}$ written in the text (4.33). 


\section{References}

[1] M. Bershadsky, S. Cecotti, H. Ooguri and C. Vafa, "Kodaira-Spencer theory of gravity and exact results for quantum string amplitudes," Commun. Math. Phys. 165, 311 (1994) arXiv:hep-th/9309140.

[2] N. Drukker and D. J. Gross, "An Exact Prediction Of N = 4 Susym Theory For String Theory," J. Math. Phys. 42, 2896 (2001) arXiv:hep-th/0010274.

[3] S. J. Rey and J. T. Yee, "Macroscopic strings as heavy quarks in large N gauge theory and anti-de Sitter supergravity," Eur. Phys. J. C 22, 379 (2001) arXiv:hepth/9803001].

[4] J. M. Maldacena, "Wilson loops in large N field theories," Phys. Rev. Lett. 80, 4859 (1998) arXiv:hep-th/9803002.

[5] N. Drukker, D. J. Gross and H. Ooguri, "Wilson loops and minimal surfaces," Phys. Rev. D 60, 125006 (1999) arXiv:hep-th/9904191.

[6] J. K. Erickson, G. W. Semenoff and K. Zarembo, "Wilson loops in N = 4 supersymmetric Yang-Mills theory," Nucl. Phys. B 582, 155 (2000) arXiv:hep-th/0003055.

[7] M. Bianchi, M. B. Green and S. Kovacs, "Instanton corrections to circular Wilson loops in N = 4 supersymmetric Yang-Mills," JHEP 0204, 040 (2002) arXiv:hepth/0202003.

[8] G. 't Hooft, "A Planar Diagram Theory For Strong Interactions," Nucl. Phys. B 72, 461 (1974).

[9] H. Ooguri and C. Vafa, "Worldsheet Derivation Of A Large N Duality," Nucl. Phys. B 641, 3 (2002) arXiv:hep-th/0205297.

[10] J. M. Maldacena, "The large N limit of superconformal field theories and supergravity," Adv. Theor. Math. Phys. 2, 231 (1998) [Int. J. Theor. Phys. 38, 1113 (1999)] arXiv:hep-th/9711200.

[11] O. Aharony, S. S. Gubser, J. M. Maldacena, H. Ooguri and Y. Oz, "Large N field theories, string theory and gravity," Phys. Rept. 323, 183 (2000) arXiv:hep-th/9905111.

[12] H. Buchholz, The Confluent Hypergeometric Function (1969), Springer-Verlag.

[13] J. Abad and J. Sesma, "Computation of the Regular Confluent Hypergeometric Function," Mathematica Journal 5, 4 (1995).

[14] N. Drukker and B. Fiol, "All-genus calculation of Wilson loops using D-branes," JHEP 0502, 010 (2005) arXiv:hep-th/0501109.

[15] E. Witten, "Quantum field theory and the Jones polynomial," Commun. Math. Phys. 121, 351 (1989).

[16] R. Gopakumar and C. Vafa, "On the gauge theory/geometry correspondence," Adv. Theor. Math. Phys. 3, 1415 (1999) arXiv:hep-th/9811131.

[17] M. Marino, "Chern-Simons Theory, Matrix Integrals, And Perturbative ThreeManifold Invariants," Commun. Math. Phys. 253, 25 (2004) arXiv:hep-th/0207096.

[18] M. Aganagic, A. Klemm, M. Marino and C. Vafa, "Matrix model as a mirror of Chern-Simons theory," JHEP 0402, 010 (2004) arXiv:hep-th/0211098. 